\title{
NETWORK DESIGN OF INTEGRATED E-SUPPLY CHAIN FOR AGILE MANUFACTURING
}

\author{
M. Dotoli*, M.P. Fanti*, C. Meloni*, M.C. Zhou** \\ *Dipartimento di Elettrotecnica ed Elettronica, Politecnico di Bari, Bari Italy \\ **Dep. of Electrical and Computer Engineering, New Jersey Institute of Technology, Newark, NJ, USA
}

\begin{abstract}
Supply chains are distributed manufacturing systems composed of various resources belonging to different companies. The configuration of the supply chain network, integrated with Internet and web-based electronic market places, is essential for business to pursue a competitive advantage. This paper develops a model describing the integrated e-supply chain network and formulates a multi-criteria integer linear optimization problem to select the candidates and the links connecting the stages of the e-supply chain. Hence, some multi-criteria objective functions are defined and suited constraints are introduced on the basis of the digraph modelling the supply chain network. The proposed methodology is illustrated by way of a case study describing a network for desktop computer production. Copyright ( 2005 IFAC
\end{abstract}

Keywords: manufacturing systems, agile manufacturing, networks, optimization, performance indices.

\section{INTRODUCTION}

Agile manufacturing has been introduced as a concept to satisfy the demand for low volume and high variety products (Wu et al., 1999, Yang et al., 2003). By integrating computer systems, hardware and information flows, automated manufacturing systems provide agile manufactures with flexibility and reconfigurability (Luo et al., 2001). Moreover, a common and accepted issue to achieve agility in a manufacturing system is manufacturing products in geographically different sites connected through communication networks (Wu et al., 1999). In other words, the manufacturing system is composed of various resources belonging to different companies that rise to the formation of a Supply Chain (SC) network. A SC network is defined as a collection of independent companies, possessing complementary skills and integrated with streamlined material, information and financial flow (Viswanadham and Gaonkar, 2003). The core of highly competitive and efficient SC networks is collaboration: Internet and web-based electronic market places can provide an inexpensive, secure and pervasive medium for information transfer between business units. Hence, Integrated E-Supply Chain (IESC) is an emerging business strategy that integrates the supply chain design and the power of e-commerce in order to obtain more flexible and agile manufacturing processes. In the formation of an effective dynamic IESC network, the selection of partners in each tier of the SC for fulfillment of each and every order is extremely important (Viswanadham and Gaonkar, 2003). Significant literature deals with the problem of SC component selection and network design (Gaonkar and Viswanadham, 2001, Jang et al., 2002, Luo et al. 2001, Viswanadham and Gaonkar, 2003, Wu et al., 1999) to achieve agility and flexibility. In particular, Luo et al. (2001) present a novel approach to model and optimize an IESC network that incorporates e-commerce and electronic linkage. Interaction and tradeoffs occurring between the network components are analyzed and optimized using a fuzzy multi-objective optimization approach. This paper starts from the network model proposed by Luo et al. (2001) and proposes a configuration strategy for IESC network design, considering also the e-business relationships between operators and the network environmental impact. The structure of the IESC is described by a digraph where nodes are partners and edges are links. Different costs are assigned to each link (edge) so that the performance indices can be obtained by the digraph structure. The considered data are transportation and information 
connections among stages, costs and transport pollution. A multi-criteria integer linear optimization problem is formulated to configure the IESC network. Hence, single criterion and multi-criteria objective functions are defined and suited constraints are introduced and determined on the basis of the digraph structure. A case study is analyzed and different solutions are presented and compared to show the flexibility of the proposed decision strategy approach.

This paper is organized as follows. Section 2 recalls the network model employing digraphs and section 3 defines the optimization model and the constraints. In section 4 a case study is analyzed and solved. Finally, section 5 summarizes the conclusions.

\section{THE NETWORK MODEL}

\subsection{The Integrated E-Supply Chain Description}

An Integrated E-Supply Chain (IESC) network can be defined as a hyper-network of material flows overlaid with an e-business information network. The considered IESC contains different stages: raw material supply, intermediate supply, manufacturing, distribution, retail, customers, and de-manufacturing or re-cycling. For example, we consider inbound stages in which partners are raw material suppliers or plant stages. Moreover, the distribution stages have partners such as manufacturers, product distributions and warehouses. Finally, outbound stages can be composed of retailers, customers, recyclers and demanufacturers. After the de-manufacturing stage, recovered material, components or energy feedback to suitable supply chain stages are considered. We denote the IESC stages by the set $\mathbf{S T}=\left\{\mathbf{P}_{1}, \ldots, \mathbf{P}_{\mathbf{k}}, \ldots, \mathbf{P}_{\mathbf{N}_{\mathbf{s}}}\right\}$, where $\mathrm{N}_{\mathrm{S}}$ is the number of stages. In particular, each stage $\mathbf{P}_{\mathbf{k}}$ is described as a set of $s_{\mathrm{k}}$ partners representing different actors of the

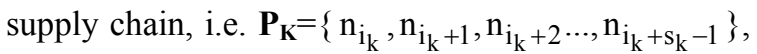
where $i_{k}$ is the generic index such that $i_{k}=\sum_{h}^{k-1} s_{h}$ with $\mathrm{k}=2,3 \ldots, \mathrm{N}_{\mathrm{s}}$ and $\mathrm{i}_{1}=1$. We suppose that there are $\mathrm{N}$ partners in the system. Moreover, the Bill Of Material (BOM) of stage $\mathbf{P}_{\mathbf{k}}$ is a set of material and components required for processes in the $\mathrm{k}$-th stage and produced by upstream stages.

To describe the interactions among partners of different stages of an IESC, two sets of links are introduced: material flow links ( $m$-links) and information links (e-links). While a traditional SC only exhibits $m$-links that describe the flow and transport of materials, in an IESC also $e$-links are considered. This kind of links describe the connection among stages realized by Internet and allow direct and fast flow of material between two non-consecutive stages. The $m$-links of stage $\mathbf{P}_{\mathbf{k}}$ are denoted by the set $\mathbf{L}_{\mathbf{m}}=\left\{\mathrm{m}_{\mathrm{ij}}\right\}$, where $\mathrm{m}_{\mathrm{ij}}$ is an $m$-link starting from $\mathrm{n}_{\mathrm{i}} \in \mathbf{P}_{\mathbf{k}}$ and ending in $\mathrm{n}_{\mathrm{j}} \in \mathbf{P}_{\mathbf{h}}$, with $\mathbf{P}_{\mathbf{k}}, \mathbf{P}_{\mathbf{h}} \in \mathbf{S T}$ and $\mathrm{k} \neq \mathrm{h}$. Analogously, we define the $e$ link set as $\mathbf{L}_{\mathbf{e}}=\left\{\mathrm{e}_{\mathrm{ij}}\right\}$, where $\mathrm{e}_{\mathrm{ij}}$ is an $e$-link starting from $\mathrm{n}_{\mathrm{i}} \in \mathbf{P}_{\mathbf{k}}$ and ending to $\mathrm{n}_{\mathrm{j}} \in \mathbf{P}_{\mathbf{h}}$, with $\mathbf{P}_{\mathbf{k}}, \mathbf{P}_{\mathbf{h}} \in \mathbf{S T}$ and $\mathrm{k} \neq \mathrm{h}$. The set $\mathbf{L}=\mathbf{L}_{\mathbf{m}} \cup \mathbf{L}_{\mathbf{e}}$ denotes the complete set of links of the IESC. We assume that two partners can be connected by $m$-links and/or by $e$-links. We remark that IESC partners belonging to the same stage are not connected by links. Indeed, in the considered model material and information flow through different stages. Fig. 1 depicts a generic IESC network.

We conclude the description of the SC network by introducing the set of performance indices $\mathbf{M}=\left\{\mathrm{M}_{1}, \mathrm{M}_{2}, \ldots, \mathrm{M}_{\mathrm{N}_{\mathrm{M}}}\right\}$, where each element $\mathrm{M}_{\mathrm{q}} \in \mathbf{M}$ corresponds to a performance measure. Typical indices include cost, cycle time, product quality, energy consumption and environmental impact (Beamon, 1999, Luo et al., 2001). Hence, a performance value is assigned to each link, considering $m$ - and $e$-links: $\mathrm{M}_{\mathrm{q}}\left(\mathrm{m}_{\mathrm{ij}}\right)\left(\mathrm{M}_{\mathrm{q}}\left(\mathrm{e}_{\mathrm{ij}}\right)\right)$ with $\mathrm{q}=1, \ldots, \mathrm{N}_{\mathrm{M}}$ denotes the value of the performance measure $\mathrm{M}_{\mathrm{q}}$ associated with the link $\mathrm{m}_{\mathrm{ij}} \in \mathbf{L}_{\mathbf{m}}\left(\mathrm{e}_{\mathrm{ij}} \in \mathbf{L}_{\mathbf{e}}\right)$. These performance indices encapsulate the characteristics and the properties of each link. At this point an objective function has to be defined to select the partners of the IESC network and the links connecting the stages.

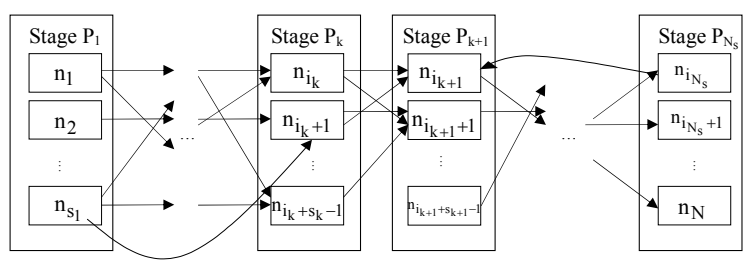

Fig. 1. The structure of a generic IESC network.

The case study. We consider a case study inspired by an example proposed in Luo et al. (2001). The target product is a typical desktop computer system consisting of the computer, hard disk driver, monitor, keyboard and mouse. The $\mathrm{SC}$ is composed of $\mathrm{N}_{\mathrm{S}}=6$ stages: four suppliers, one manufacturer, two distributors, two retailers, one consumer and four recyclers, for a total of $\mathrm{N}=14$ partners. Fig. 2 shows the IESC structure before the IESC configuration and optimization are performed. The network exhibits the $e$-links $\mathrm{e}_{18}, \mathrm{e}_{17}$ and $\mathrm{e}_{4,10}$, the $m$ - and $e$-links $\mathrm{m}_{78}, \mathrm{e}_{78}$, $\mathrm{m}_{79}, \mathrm{e}_{79}, \mathrm{~m}_{7,10}, \mathrm{e}_{7,10}, \mathrm{~m}_{9,10}, \mathrm{e}_{9,10}, \mathrm{~m}_{8,10}$, and $\mathrm{e}_{8,10}$, while the remaining links are $m$-links. In particular, the considered e-links model the information flow associated to electronic commerce. For example, $\mathrm{e}_{18}$, $\mathrm{e}_{17}$ and $\mathrm{e}_{4,10}$ model the $e$-business practice in which a supplier ships components or products directly to distributors or consumers; similarly, partners $\mathrm{n}_{7}, \mathrm{n}_{8}$ and $\mathrm{n}_{9}$ run an $e$-store together with a traditional store, so that their output links are characterized both by material and information flow. The data for the case study are reported in Table 1 (Luo et al., 2001), that shows the values of each performance index $\mathrm{M}_{\mathrm{q}}$ with $\mathrm{q}=1,2$ and 3 associated with the links of the considered IESC, determined on the basis of industrial experience. More precisely, the adopted performance indices are total costs $\left(\mathrm{M}_{1}\right), \mathrm{CO}_{2}$ emission $\left(\mathrm{M}_{2}\right)$ and energy $\left(\mathrm{M}_{3}\right)$. Moreover, note that rows in Table 1 referring to $e$-links only exhibit low value indices, due to the low costs associated to electronic transactions. 


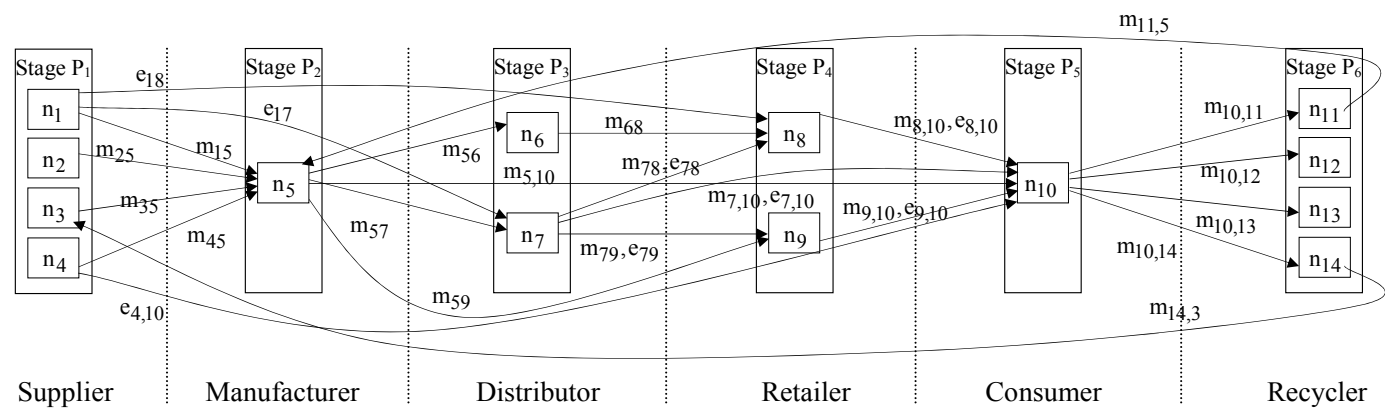

Fig. 2. The stages of the IECS network for the case study.

Table 1. Data sheet for the case study network links

\begin{tabular}{lccc} 
Links & $\begin{array}{c}\text { Total costs } \\
\left(\mathrm{M}_{1}\right)\end{array}$ & $\begin{array}{c}\text { CO2 emission } \\
\left(\mathrm{M}_{2}\right)\end{array}$ & $\begin{array}{c}\text { Energy } \\
(\mathrm{M} 3)\end{array}$ \\
\hline $\mathrm{e}_{18}\left(\mathrm{x}_{1}\right)$ & 41.80 & 0.87 & 359.00 \\
$\mathrm{e}_{17}\left(\mathrm{x}_{2}\right)$ & 46.70 & 0.74 & 332.00 \\
$\mathrm{~m}_{15}\left(\mathrm{x}_{3}\right)$ & 319.00 & 2.21 & 1479.00 \\
$\mathrm{~m}_{25}\left(\mathrm{x}_{4}\right)$ & 308.00 & 2.19 & 1776.00 \\
$\mathrm{~m}_{35}\left(\mathrm{x}_{5}\right)$ & 238.00 & 3.10 & 1540.00 \\
$\mathrm{~m}_{45}\left(\mathrm{x}_{6}\right)$ & 246.00 & 1.47 & 1409.00 \\
$\mathrm{e}_{4,10}\left(\mathrm{x}_{7}\right)$ & 53.90 & 30.20 & 369.00 \\
$\mathrm{~m}_{56}\left(\mathrm{x}_{8}\right)$ & 448.00 & 8.74 & 3618.00 \\
$\mathrm{~m}_{5,10}\left(\mathrm{x}_{9}\right)$ & 379.00 & 296.00 & 3542.00 \\
$\mathrm{~m}_{57}\left(\mathrm{x}_{10}\right)$ & 358.00 & 6.26 & 2885.00 \\
$\mathrm{~m}_{59}\left(\mathrm{x}_{11}\right)$ & 358.00 & 223.00 & 3259.00 \\
$\mathrm{~m}_{68}\left(\mathrm{x}_{12}\right)$ & 20.89 & 0.87 & 13.40 \\
$\mathrm{~m}_{78}, \mathrm{e}_{78}\left(\mathrm{x}_{13}\right)$ & 25.20 & 1.10 & 16.40 \\
$\mathrm{~m}_{7,10}, \mathrm{e}_{7,10}\left(\mathrm{x}_{14}\right)$ & 22.90 & 2.58 & 35.10 \\
$\mathrm{~m}_{79}, \mathrm{e}_{79}\left(\mathrm{x}_{15}\right)$ & 20.70 & 0.59 & 9.18 \\
$\mathrm{~m}_{8,10}, \mathrm{e}_{8,10}\left(\mathrm{x}_{16}\right)$ & 64.00 & 0.56 & 90.40 \\
$\mathrm{~m}_{9,10}, \mathrm{e}_{9,10}\left(\mathrm{x}_{17}\right)$ & 58.10 & 0.13 & 4.68 \\
$\mathrm{~m}_{10,11}\left(\mathrm{x}_{18}\right)$ & 0.42 & 0.37 & 4.80 \\
$\mathrm{~m}_{10,12}\left(\mathrm{x}_{19}\right)$ & 0.42 & 0.37 & 4.80 \\
$\mathrm{~m}_{10,13}\left(\mathrm{x}_{20}\right)$ & 0.42 & 0.37 & 4.80 \\
$\mathrm{~m}_{10,14}\left(\mathrm{x}_{21}\right)$ & 0.42 & 0.37 & 4.80 \\
$\mathrm{~m}_{11,5}\left(\mathrm{x}_{22}\right)$ & -18.00 & 0.74 & -11.00 \\
$\mathrm{~m}_{14,3}\left(\mathrm{x}_{23}\right)$ & -28.00 & 1.10 & -6.60 \\
\hline & & & \\
\hline
\end{tabular}

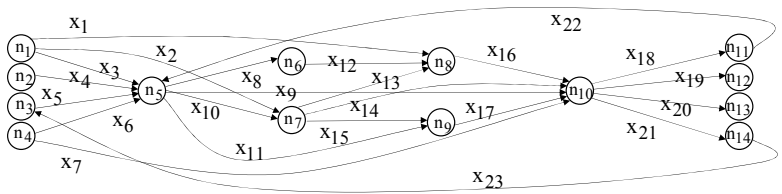

Fig. 3. The digraph associated with the case study.

\subsection{The digraph definition}

To exhibit the interactions among the stages of the IESC, we define a direct graph $\mathbf{D}=(\mathbf{N}, \mathbf{E})$. The node set $\mathbf{N}$ represents the partner set of the network and each node $n_{i} \in \mathbf{N}$ for $i=1, \ldots, N$ is associated with partner $n_{i} \in \mathbf{P}_{\mathbf{k}}$ for $k \in\left\{1, \ldots, N_{S}\right\}$ of the SC network. For the sake of simplicity, the same symbols indicate nodes and partners. Moreover, the edge set $\mathbf{E}$ is such that an arc $x_{h}$ directed from $n_{i}$ to $n_{j}$ is in $\mathbf{E}$ if there exists an $m$-link $\mathrm{m}_{\mathrm{ij}} \in \mathbf{L}_{\mathbf{m}}$ and/or an $e$-link $\mathrm{e}_{\mathrm{ij}} \in \mathbf{L}_{\mathbf{e}}$. We denote with $\mathrm{E}$ the number of edges in $\mathbf{D}$.

The case study (continued): the associated digraph. Fig. 3 depicts the digraph describing the IESC for the case study. The digraph $\mathbf{D}=(\mathbf{N}, \mathbf{E})$ has $\mathrm{N}=14$ nodes and $\mathrm{E}=23$ edges. Edges $\mathrm{x}_{1}, \mathrm{x}_{2}, \mathrm{x}_{7}$ are associated with $e$-links only, edges $\mathrm{x}_{13}, \mathrm{x}_{14}, \mathrm{x}_{15}, \mathrm{x}_{16}$ and $\mathrm{x}_{17}$ are associated both with $m$ - and $e$-links and the remaining edges of the digraphs are associated with $m$-links only (see Table 1).

\section{THE OPTIMIZATION MODEL}

Having defined the digraph depicting the interactions among the stages of the IESC, it is necessary to develop the optimization models for partner and link selection making use of available information on costs, $\mathrm{CO}_{2}$ emission and energy. The mathematical model is based on the combinatorial characteristics of the IESC and an Integer Linear Programming (ILP) problem is obtained. More precisely, the aim of the model is to minimize a single-criterion or multicriteria cost function subject to a set of constraints that we characterize as BOM, path, mutual exclusion and structural constraints. The objective function and constraints are obtained on the basis of the analysis of the digraph describing the IESC structure.

Let us consider the performance index $\mathrm{M}_{\mathrm{q}}\left(\mathrm{m}_{\mathrm{ij}}\right)$ $\left(\mathrm{M}_{\mathrm{q}}\left(\mathrm{e}_{\mathrm{ij}}\right)\right)$ that is associated with link $\mathrm{m}_{\mathrm{ij}}\left(\mathrm{e}_{\mathrm{ij}}\right)$ and with the corresponding edge $\mathrm{x}_{\mathrm{h}} \in \mathbf{E}$ connecting $\mathrm{n}_{\mathrm{i}} \in \mathbf{P}_{\mathbf{k}}$ to $\mathrm{n}_{\mathrm{j}} \in \mathbf{P}_{\mathbf{h}}$. Let us indicate with $\mathbf{c}^{\mathrm{q}}=\left[\begin{array}{llll}\mathrm{c}^{\mathrm{q}}{ }_{1} & \mathrm{c}^{\mathrm{q}}{ }_{2} & \ldots & \mathrm{c}^{\mathrm{q}}{ }_{\mathrm{E}}\end{array}\right]^{\mathrm{T}}$ the vector of $E$ entries where the h-th entry is $c^{q}{ }_{h}=M_{q}\left(e_{i j}\right)$ and/or $c^{q}{ }_{h}=M_{q}\left(m_{i j}\right)$ associated with edge $x_{h} \in \mathbf{E}$. Moreover, we denote by $\mathbf{x}=\left[\begin{array}{llll}\mathrm{x}_{1} & \mathrm{x}_{2} & \ldots \mathrm{x}_{\mathrm{E}}\end{array}\right]^{\mathrm{T}}$ an integer vector where each element $x_{h} \in\{0,1\}$ with $h=1, \ldots, E$ indicates the presence $\left(x_{h}=1\right)$ or the absence $\left(x_{h}=0\right)$ of the link $x_{h} \in \mathbf{E}$ connecting $n_{i} \in \mathbf{P}_{\mathbf{k}}$ to $n_{j} \in \mathbf{P}_{\mathbf{h}}$ in the IESC structure. The optimization problem is as follows:

$$
\mathrm{z}=\min \mathbf{f}(\mathbf{x})
$$

subject to

$$
\mathbf{A x} \geq \mathbf{B}
$$

$$
\mathrm{x}_{\mathrm{h}} \in\{0,1\} \text { for } \mathrm{h}=1, \ldots, \mathrm{E}
$$

where $\mathbf{A}$ is the constraint matrix of dimension $\mathrm{v} \times \mathrm{E}$ and $\mathbf{B}$ is a v-vector of integers, $\mathbf{v}$ representing the number of constraints. Minimizing the objective function $\mathbf{f}(\mathbf{x})$ means either to minimize only one performance index (problem 1) or a subset of the chosen performance indices (problem 2).

\subsection{The objective function}

Problem 1. The single-criterion objective function is defined as follows:

$$
\mathbf{f}(\mathbf{x})=\left(\mathbf{c}^{\mathbf{q}}\right)^{\mathrm{T}} \mathbf{x}
$$


Each solution $\mathbf{x}^{*}$ of the ILP (1)-(4) for a particular vector $\mathbf{c}^{\mathbf{q}}$ corresponds to a possible IESC structure. More precisely, the optimal solution vector $\mathbf{x}^{*}$ selects a sub-digraph $\mathbf{D}^{*}=\left(\mathbf{N}^{*}, \mathbf{E}^{*}\right)$ of $\mathbf{D}$ where $\mathbf{N}^{*} \subset \mathbf{N}$ and $\mathbf{E}^{*} \subset \mathbf{E}$, respectively. If the h-th entry of $\mathbf{x}^{*}$ is $\mathrm{X}^{*}{ }_{\mathrm{h}}=1$ and $x_{h}$ is an edge outgoing from $n_{i} \in \mathbf{P}_{\mathbf{k}}$ and incoming to $\mathrm{n}_{\mathrm{j}} \in \mathbf{P}_{\mathrm{h}}$, then the solution selects the edges $\mathrm{x}_{\mathrm{h}} \in \mathbf{E}^{*}$ and the nodes $n_{i}, n_{j} \in \mathbf{N}^{*}$. In other words, the optimal IESC with respect to index $\mathrm{M}_{\mathrm{q}}$ is described by subdigraph $\mathbf{D}^{*}$ that exhibits the actors (nodes) and the links (edges) selected in the IESC design.

Problem 2.The multi-objective function is defined as follows:

$$
\mathbf{f}(\mathbf{x})=\mathbf{C x}
$$

where $\mathbf{C}=\left[\begin{array}{c}\left(\mathbf{c}^{\mathrm{q} 1}\right)^{\mathrm{T}} \\ \cdot \\ \left(\mathrm{c}^{\mathrm{qQ}}\right)^{\mathrm{T}}\end{array}\right]$ is a $\mathrm{q}_{\mathrm{Q}} \times \mathrm{E}$ criteria matrix and $\mathbf{c}^{\mathbf{q} \mathbf{1}}, \ldots, \mathbf{c}^{\mathbf{q Q}}$ are vectors associated with performance indices $\mathrm{M}_{\mathrm{q} 1}, . ., \mathrm{M}_{\mathrm{qQ}}$, respectively.

The multi-criteria linear program solving (1)-(3) and (5) provides the maximal Pareto face of the solutions set (Ehrgott, 2000). More precisely, we obtain a subset of solutions $\mathbf{X}^{*}=\left\{\mathbf{X}^{*}\right\}$ where each $\mathbf{x}^{*}{ }_{\mathbf{i}} \in \mathbf{X}^{*}$ is a Pareto optimal solution corresponding to a subdigraph $\mathbf{D} *_{i}$ of $\mathbf{D}$ and to an IESC structure.

\subsection{Constraint Definition}

BOM constraints. Each supplier candidate can provide a subset of materials (or components) to each producer. Analogously, the retailer actors provide a subset of products to each consumer. Generally, the BOM of each partner of the manufacturer or consumer stage is described as a list of materials needed by the stage. The actor of the considered stage $\mathbf{P}_{\mathbf{k}}$ (for example, manufacturer or consumer) has to obtain all the BOM components. For example, let us suppose that the manufacturer requires all the components necessary to assemble the final product. In addition, suppose that each of these products can be shipped by means of edges $\mathrm{x}_{1}$ or $\mathrm{x}_{2}$ or $\mathrm{x}_{3}$. This condition can be written as an inequality in $0-1$ variables: $x_{1}+x_{2}+x_{3} \geq 1$. Hence, if we suppose that there are $\mathrm{v}_{1}$ BOM constraints, then the following inequality constraints are formulated:

$$
\mathbf{A}_{1} \mathbf{x} \geq \mathbf{1}
$$

where $\mathbf{1}$ is a $\mathrm{v}_{1}$-entry vector with all elements equal to 1 and $\mathbf{A}_{1}$ is a $\mathrm{v}_{1} \times \mathrm{E}$ constraint matrix with elements $A_{1}(i, j) \in\{0,1\}$ for $j=1, \ldots, E$ and $i=1, \ldots, v_{1}$.

Path constraints. It is necessary to select in the digraph at least a path starting from a node of the producers and ending to the nodes of the consumers. To impose this condition, we associate with the digraph the $\mathrm{N} \times \mathrm{E}$ incidence matrix $\mathbf{I}_{\mathbf{M}}$, where each element is $\mathbf{I}_{\mathbf{M}}(\mathrm{i}, \mathrm{j}) \in\{-1,0,1\}$. More precisely: $\mathbf{I}_{\mathbf{M}}(\mathrm{i}, \mathrm{j})=0$ if $\operatorname{arc} \mathrm{x}_{\mathrm{j}}$ does not belong to node $\mathrm{n}_{\mathrm{i}}$, $\mathbf{I}_{\mathbf{M}}(\mathrm{i}, \mathrm{j})=-1$ if $\operatorname{arc} \mathrm{x}_{\mathrm{j}}$ starts from node $\mathrm{n}_{\mathrm{i}}$, $\mathbf{I}_{\mathbf{M}}(\mathrm{i}, \mathrm{j})=1$ if $\operatorname{arc} \mathrm{x}_{\mathrm{j}}$ ends at node $\mathrm{n}_{\mathrm{i}}$.
Moreover, to define a constraint that imposes the presence of a path starting from node $n_{h}$ and ending in node $\mathrm{n}_{\mathrm{W}}$, we introduce the N-vector $\mathbf{b}_{\mathbf{h}, \mathbf{w}}=\left[\mathrm{b}_{1} \mathrm{~b}_{2} \ldots\right.$ $\left.b_{N}\right]^{T}$ with $b_{h}=-1, b_{W}=1$ and $b_{p}=0$ for $p \neq h, w$ and $\mathrm{p}=1, \ldots, \mathrm{N}$. The constraint is written as:

$$
\mathbf{I}_{\mathbf{M}} \mathbf{x} \geq \mathbf{b}_{\mathbf{h}, \mathbf{w}} \text {. }
$$

Hence, the constraint sub-matrix and the left side vector are:

$$
\mathbf{A}_{2}=\left[\begin{array}{c}
I_{M} \\
\cdots \\
I_{M}
\end{array}\right] \text { and } \mathbf{B}_{2}=\left[\begin{array}{c}
b_{h 1, w 1} \\
\ldots \\
b_{h i, w i}
\end{array}\right] .
$$

Mutual exclusion constraints. In some cases it is necessary to choose only one actor in a stage. In other words, the condition "only one edge among $\mathrm{x}_{1}$, $\mathrm{x}_{2}$ and $\mathrm{x}_{3}$ can be in the solution digraph" is written with the following inequality in $0-1$ variables: $\mathrm{x}_{1}+\mathrm{x}_{2}+\mathrm{x}_{3} \leq 1$. On the other hand, the condition "one and only one edge among $\mathrm{x}_{1}, \mathrm{x}_{2}$ and $\mathrm{x}_{3}$ has to be in the solution digraph" is expressed by $x_{1}+x_{2}+x_{3}=1$. Hence, $v_{3}$ mutual exclusion constraints can be expressed by the following equation:

$$
-A_{3} x \geq-1
$$

where $\mathbf{1}$ is a $v_{3}$-entry vector with all elements equal to 1 and matrix $\mathbf{A}_{\mathbf{3}}$ is a constraint matrix with $A_{3}(i, j) \in\{0,1\}$ for $j=1, \ldots, E$ and $i=1, \ldots, v_{3}$.

Structural constraints. Some particular structural constraints are related to the digraph. For example, the following condition can be imposed: "if edge $\mathrm{x}_{1}$ belongs to the solution digraph then edges $\mathrm{x}_{2}$ or $\mathrm{x}_{3}$ belong to the solution digraph". This condition is expressed by the constraint: $x_{2}+x_{3} \geq x_{1}$. A second type of structural condition is: " $\mathrm{x}_{2}$ belongs to the solution digraph if and only if (iff) $\mathrm{x}_{1}$ belongs to the solution digraph", i.e., $\mathrm{x}_{2}=\mathrm{x}_{1}$. Therefore, the $\mathrm{v}_{4}$ structural constraints can be expressed as follows:

$$
\mathbf{A}_{\mathbf{4}} \mathbf{x} \geq \mathbf{0}
$$

with $A_{4}(i, j) \in\{0,1,-1\}$ for $j=1, \ldots, E$ and $i=1, \ldots, v_{4}$.

Finally, the constraints matrix and specification vector are the following:

$$
\mathbf{A}=\left[\begin{array}{c}
\mathbf{A}_{1} \\
\mathbf{A}_{2} \\
-\mathbf{A}_{3} \\
\mathbf{A}_{4}
\end{array}\right] \text { and } \mathbf{B}=\left[\begin{array}{c}
\mathbf{1} \\
\mathbf{B}_{2} \\
-1 \\
\mathbf{0}
\end{array}\right] \text {. }
$$

\section{CASE Study}

To illustrate the network design procedure, the case study described in Sections 2, Table 1 and Fig. 2 and 3 is considered. Various computational experiments are performed minimizing considering cost, $\mathrm{CO}_{2}$ emission and energy consumption, alternatively. Furthermore, three multi-objective functions for problem 2 are chosen. Solutions are obtained by the well-known two-phases simplex method in the Matlab framework (Venkataraman, 2001). 


\subsection{Constraint Definition}

BOM constraints. The components supplier constraints are obtained taking into account that the BOM of the second stages in Fig. 3, representing the manufacturer, is as follows: computer $(\mathrm{C})$, hard-diskdriver $(\mathrm{H})$, monitor $(\mathrm{M})$, and keyboard/mouse $(\mathrm{K})$. We assume that $C$ is produced by $n_{1}$ and $n_{2}, H$ is produced by $\mathrm{n}_{1}, \mathrm{n}_{2}$ and $\mathrm{n}_{3}, M$ is produced by $\mathrm{n}_{2}, \mathrm{n}_{3}$ and $\mathrm{n}_{4}$, and $\mathrm{K}$ is produced by $\mathrm{n}_{3}$ and $\mathrm{n}_{4}$ (Luo et al., 2001). Hence, with reference to Fig. 3 the constraints imposed on the edges are as follows:

$$
\begin{aligned}
& \mathrm{x}_{3}+\mathrm{x}_{4} \geq 1 \\
& \mathrm{x}_{3}+\mathrm{x}_{4}+\mathrm{x}_{5} \geq 1 \\
& \mathrm{x}_{4}+\mathrm{x}_{5}+\mathrm{x}_{6} \geq 1 \\
& \mathrm{x}_{5}+\mathrm{x}_{6} \geq 1
\end{aligned}
$$

Path constraints. Since the network of the case study includes one manufacturer and one consumer only, a path between node $\mathrm{n}_{5}$ and $\mathrm{n}_{10}$ is needed. Consequently, we build the $\mathrm{N} \times \mathrm{E}$ incidence matrix $\mathbf{I}_{\mathbf{M}}$ associated with digraph $\mathbf{D}$. Moreover, we define the 23-vector $\mathbf{b}_{5,10}=\left[\begin{array}{llll}b_{1} & b_{2} & \ldots & b_{23}\end{array}\right]$ with $b_{5}=-1 \quad b_{10}=1$ and other $b_{p}$ 's equal 0 . The constraint that imposes the presence of a path starting from node $n_{5}$ and ending at node $\mathrm{n}_{10}$ is written as follows:

$$
\mathbf{I}_{\mathbf{M}} \mathbf{x} \geq \mathbf{b}_{5,10}
$$

Mutual exclusion constraints. It is assumed that the design conditions of the SC network include the hypothesis that one and only one partner is to be present in the recycler stage (stage $\mathrm{P}_{6}$ in Fig. 2). Furthermore, only one type of commerce is present between the second and third stages and one and only one e-link is present among the first stage and the others. Hence, with reference to Fig. 3, the mutual exclusion constraints are as follows:

$$
\begin{aligned}
& \mathrm{x}_{18}+\mathrm{x}_{19}+\mathrm{x}_{20}+\mathrm{x}_{21} \leq 1 \\
& \mathrm{x}_{13}+\mathrm{x}_{14}+\mathrm{x}_{15} \leq 1 \\
& \mathrm{x}_{1}+\mathrm{x}_{2}+\mathrm{x}_{7}=1
\end{aligned}
$$

Structural constraints. The constraints derived from the digraph structure are as follows:

$$
\begin{aligned}
& x_{22}-x_{18}=0 \\
& x_{23}-x_{21}=0 \\
& x_{5}-x_{23} \geq 0 \\
& x_{16}-x_{1} \geq 0 \\
& x_{13}+x_{14}+x_{15}-x_{2} \geq 0 \\
& x_{16}-x_{13} \geq 0 \\
& x_{17}-x_{15} \geq 0
\end{aligned}
$$

Hence, the first line of (15) means that $x_{22}$ is selected iff $\mathrm{x}_{18}$ is selected. In addition, the third line of (15) means that if $x_{23}$ is selected then $x_{5}$ is selected.

\subsection{Solution of Problem 1}

Problem 1 is solved considering three different performance indices: costs $\left(\mathrm{M}_{1}\right)$, energy $\left(\mathrm{M}_{2}\right)$ and $\mathrm{CO}_{2}$ emission $\left(\mathrm{M}_{3}\right)$. The corresponding objective functions are denoted $\mathbf{f}_{\mathbf{1}}, \mathbf{f}_{\mathbf{2}}$ and $\mathbf{f}_{\mathbf{3}}$, respectively. The obtained sub-digraphs are presented in Fig. 4, 5 and
6 respectively, and the corresponding objective functions are given in Table 2. Obviously, the showed digraphs are equal to the solution digraphs obtained in (Luo et al., 2001) with the fuzzy logic optimization method. We remark that both the solution digraphs minimizing costs and energy include a recycler (i.e. $\mathrm{n}_{14}$ in Fig. 4 and $\mathrm{n}_{11}$ in Fig. 5), which is missing in the network minimizing $\mathrm{CO}_{2}$ emission in Fig. 6. Indeed, including the recycling stage in the IESC results in a reduction of costs and energy (notice the negative entries in the last two rows of Table 1) but does not decrease $\mathrm{CO}_{2}$ emission.

Table 2. The values of objective functions $\mathbf{f}_{1}, \mathbf{f}_{2}$ and

\begin{tabular}{lccc}
\hline & $\underline{\mathbf{f}_{3}}$ for problem 1 of the case study. \\
\cline { 2 - 4 } & $\begin{array}{c}\text { Costs } \\
\text { in US\$ }\end{array}$ & $\begin{array}{c}\text { Energy } \\
\text { in MJ }\end{array}$ & $\begin{array}{c}\text { CO2 emission } \\
\text { in KgCE }\end{array}$ \\
\hline $\min \mathbf{f}_{\mathbf{1}}$ & $\mathbf{9 4 6 . 0}$ & 6566.3 & 16.34 \\
$\min \mathbf{f}_{\mathbf{2}}$ & 1030.9 & $\mathbf{6 1 1 2 . 7}$ & 12.51 \\
$\min \mathbf{f}_{\mathbf{3}}$ & 1037.5 & 6415.9 & $\mathbf{1 1 . 3 8}$ \\
\hline
\end{tabular}

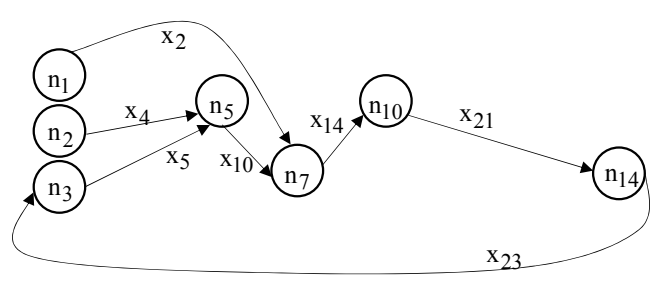

Fig. 4. Solution digraph of $\min \left(\mathrm{f}_{1}\right)$.

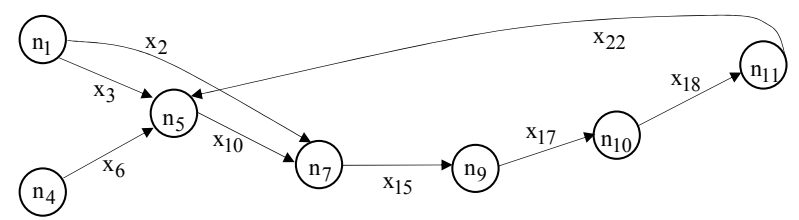

Fig. 5. Solution digraph of $\min \left(f_{2}\right)$.

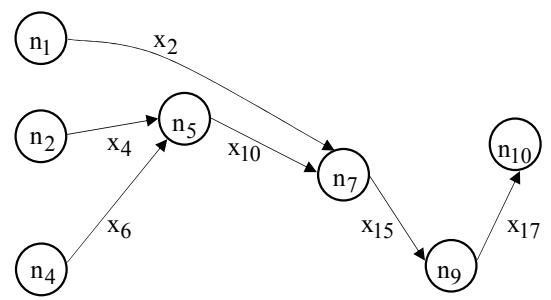

Fig. 6. Solution digraph of $\min \left(f_{3}\right)$.

\subsection{Solution of Problem 2}

The multi-objective optimization problem is solved considering the following performance indices: costs and $\mathrm{CO}_{2}$ emission $\left(\mathbf{f}_{\mathbf{4}}\right)$, costs and energy $\left(\mathbf{f}_{\mathbf{5}}\right)$, energy, cost and $\mathrm{CO}_{2}$ emission ( $\mathbf{f}_{\mathbf{6}}$ ). Tables 3, 4 and 5 report for each optimal point of the maximal Pareto faces of objective functions $\mathbf{f}_{\mathbf{4}}, \mathbf{f}_{\mathbf{5}}$ and $\mathbf{f}_{\mathbf{6}}$, the corresponding performance indices and the selected arcs of the IESC network. In particular, each solution is associated with a network with reference to the chosen multi-objective optimization problem. The digraph corresponding to solution $\mathbf{x}_{\mathbf{D}}$ in Table 5 is depicted in Fig. 7. For the sake of simplicity, we neglect representing the digraphs corresponding to the different solutions minimizing $\mathbf{f}_{\mathbf{4}}, \mathbf{f}_{\mathbf{5}}$ and $\mathbf{f}_{\mathbf{6}}$. In fact, such digraphs may easily be obtained from the last columns in Tables 3, 4 and 5, respectively. 
Table 3. The optimal solutions for multi-objective function: cost and $\mathrm{CO}_{2}$ emission $\left(\min \left(\mathbf{f}_{4}\right)\right)$.

Optimal Costs $\mathrm{CO}_{2}$ emission Indices of selected solutions in US\$ in $\mathrm{KgCE} \quad$ edges

\begin{tabular}{lccc}
\hline $\mathbf{x}_{\mathbf{A}}$ & $\mathbf{9 4 6 . 0}$ & 16.34 & $2,4,5,10,14,21,23$ \\
$\mathbf{x}_{\text {B }}$ & 964.0 & 14.35 & $2,4,6,10,14,18,22$ \\
$\mathbf{x}_{\mathbf{C}}$ & 981.6 & 13.24 & $2,4,6,10,14$ \\
$\mathbf{x}_{\mathbf{D}}$ & 1030.9 & 12.51 & $2,3,6,10,15,17,18$, \\
$\mathbf{x}_{\mathbf{E}}$ & 1037.5 & $\mathbf{1 1 . 3 8}$ & $2,4,6,10,15,17$ \\
\hline
\end{tabular}

Table 4. The optimal solutions for multi-objective function: energy and $\mathrm{CO}_{2}$ emission $\left(\min \left(\mathbf{f}_{5}\right)\right)$.

Optimal Energy $\mathrm{CO}_{2}$ emission Indices of selected

\begin{tabular}{lrcc} 
solutions & In MJ & in $\mathrm{KgCE}$ & edges \\
\hline $\mathbf{x}_{\mathbf{A}}$ & $\mathbf{6 1 1 2 . 7}$ & 12.51 & $2,3,6,10,15,17,18,22$ \\
$\mathbf{x}_{\mathbf{B}}$ & 6118.9 & 11.40 & $2,3,6,10,15,17$ \\
$\mathbf{x}_{\mathbf{C}}$ & 6415.9 & $\mathbf{1 1 . 3 8}$ & $2,4,6,10,15,17$ \\
\hline
\end{tabular}

Table 5. The optimal solutions for multi-objective function: cost, energy and $\mathrm{CO}_{2}$ emission $\left(\min \left(\mathbf{f}_{6}\right)\right)$. $\mathrm{CO}_{2}$

Optimal Costs in Energy emission Indices of selected solutions US\$ in MJ in edges

\begin{tabular}{lcccc} 
& & $\mathrm{KgCE}$ & \\
\hline $\mathbf{x}_{\mathbf{A}}$ & $\mathbf{9 4 6 . 0}$ & 6566.3 & 16.34 & $2,4,5,10,14,21,23$ \\
$\mathbf{x}_{\mathbf{B}}$ & 957.0 & 6269.3 & 16.36 & $2,3,5,10,14,21,23$ \\
$\mathbf{x}_{\mathbf{C}}$ & 964.0 & 6430.9 & 14.35 & $2,4,6,10,14,18,22$ \\
$\mathbf{x}_{\mathbf{D}}$ & 975.0 & 6133.9 & 14.37 & $2,3,6,10,14,18,22$ \\
$\mathbf{x}_{\mathbf{E}}$ & 981.6 & 6437.1 & 13.24 & $2,4,6,10,14$ \\
$\mathbf{x}_{\mathbf{F}}$ & 992.6 & 6140.1 & 13.26 & $2,3,6,10,14$ \\
$\mathbf{x}_{\mathbf{G}}$ & 1030.9 & $\mathbf{6 1 1 2 . 7}$ & 12.51 & $2,3,6,10,15,17,18,22$ \\
$\mathbf{x}_{\mathbf{H}}$ & 1048.5 & 6118.9 & 11.40 & $2,3,6,10,15,17$ \\
$\mathbf{x}_{\mathbf{I}}$ & 1037.5 & 6415.9 & $\mathbf{1 1 . 3 8}$ & $2,4,6,10,15,17$
\end{tabular}

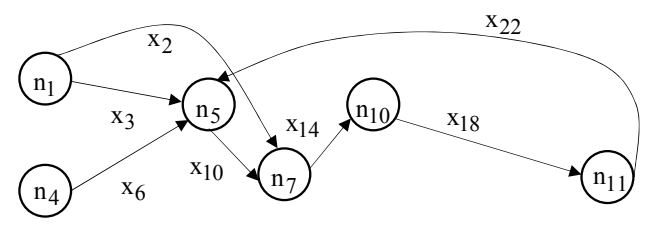

Fig. 7. Digraph representing solution $\mathbf{x}_{\mathbf{D}}$ minimizing problem 2 with objective function $\mathbf{f}_{\mathbf{6}}$.

The results show that the proposed method is able to provide a set of optimal and suboptimal solutions. For example, solutions $\mathbf{x}_{\mathbf{A}}$ and $\mathbf{x}_{\mathbf{E}}$ obtained minimizing objective function $\mathbf{f}_{\mathbf{4}}$ are correspondingly equal to the solutions obtained minimizing $\mathbf{f}_{\mathbf{1}}$ and $\mathbf{f}_{\mathbf{3}}$, respectively (see Table 3 and Fig. 4 and 6). Moreover, solutions $\mathbf{x}_{\mathbf{A}}$ and $\mathbf{x}_{\mathbf{C}}$ obtained minimizing $\mathbf{f}_{5}$ correspond to the optimization of costs and $\mathrm{CO}_{2}$ emission, respectively (see Table 4 and Fig. 5 and 6). However, in Table 3 solution $\mathbf{x}_{\mathbf{A}}$ corresponds to a large value of $\mathrm{CO}_{2}$ emission and solution $\mathbf{x}_{\mathbf{D}}$ corresponds to a large value of costs. On the contrary, solutions $\mathbf{x}_{\mathbf{B}}$ and $\mathbf{x}_{\mathbf{C}}$ of Table 3 both exhibit a good value of costs and of $\mathrm{CO}_{2}$ emission. In addition, minimizing objective function $\mathbf{f}_{6}$ we obtain solution $\mathbf{x}_{\mathbf{D}}$ in Table 5 featuring satisfactory values of costs, energy and $\mathrm{CO}_{2}$ emission. Comparing our multi-criteria methodology with the multi-criteria optimization approach of Luo et al. (2001), we point out that the fuzzy logic method provides only one solution. On the contrary, our optimization procedure enables us to choose among several near-optimal solutions preferences or priorities on the considered performance indices. This can guide the user in selecting among solutions on the same Pareto face.

\section{CONCLUSIONS}

The Integrated E-Supply Chain (IESC) is a business strategy that incorporates the power of e-commerce to streamline the manufacturing processes. An IESC system has a more complex structure than a traditional supply chain (SC) system, since it embraces the e-business strategy to establish information links and integrates end-of-life processes into the entire SC structure. This paper models the structure of the IESC by a digraph describing the actors of the stages and the material and information links among the stages. Moreover, an integer multicriteria optimization problem is stated and the procedure to obtain the constraints is drawn. The proposed methodology is applied to a case study inspired by an IESC producing desktop computers that is described in the related literature. The multicriteria optimization problem solution proposes different structures for the IESC on the basis of the performance indices and costs chosen to define the multi-criteria objective function.

\section{REFERENCES}

Beamon, B.M. (1999). Measuring supply chain performance. International Journal of Operations and Production Management, 19, pp. 257-292.

Ehrgott, M. (2000). Multicriteria Optimization. Springer-Verlag, Berlin-Heidelberg Germany.

Gaonkar, R. and N. Viswanadham (2001). Collaboration and Information Sharing in Global Contract Manufacturing Networks. IEEE/ASME Transactions on Mechatronics, 6, pp. 366-376.

Jang, Y.J., S.Y. Jeng, B.M. Chang and J. Park (2002). A combinated model of network design and production/distribution planning for a supply network. Computers \& Industrial Engineering, 43, pp. 263-281.

Luo, Y., M.C. Zhou and R.J. Caudill (2001) An integrated E-Supply Chain Model for Agile and Environmentally Conscious Manufacturing. IEEE/ASME Transactions on Mechatronics, 6 , pp. 377-386.

Venkataraman, P. (2001). Applied Optimization with MATLAB Programming. Wiley Interscience.

Viswanadham, N. and R.S.Gaonkar (2003). Partner selection and synchronized planning in dynamic manufacturing networks. IEEE Transactions on Robotics and Automation, 19, pp. 117-130.

Wu, N., N. Mao and Y. Qian (1999). An Approach to partner selection in agile manufacturing. Journal of Intelligent Manufacturing, 10, pp. 519-529.

Yang, H., Z. Yu and T.C. EdwinCheng (2003). A Strategic Model for Supply Chain design with Logical Contraints: formulation and Solution. Computers of Operations Research, 30, pp. 2135-2155 